\title{
Application of a laser produced plasma: Experimental Stark widths of single ionized lead lines
}

\author{
C. Colón
}

A. Alonso Medina

\begin{abstract}
The optical emission spectroscopy from laser produced plasma generated by a $10,640 \AA$ radiation, with an irradiance of $1.4 \times 10^{10} \mathrm{~W} \mathrm{~cm}^{-2}$ on several lead targets, in vacuum and in an atmosphere of argon, was recorded and analyzed between 1900 and $7000 \AA$. The Local Thermodynamic Equilibrium conditions and plasma homogeneity have been checked. Stark widths for 31 lines of Pb II have been measured. These lines measured in this work correspond to the transitions $7 \mathrm{~s} \rightarrow 6 \mathrm{p}, n(n=8,9,10) \mathrm{s} \rightarrow 7 \mathrm{p}, n(n=7,8) \mathrm{p} \rightarrow 7 \mathrm{~s}, n(n=7,8) \mathrm{p} \rightarrow 6 \mathrm{p}^{2}, n(n=7,8) \mathrm{d} \rightarrow 7 \mathrm{p}, n(n=5,6) \mathrm{f} \rightarrow 6 \mathrm{~d}$, $n(n=5,6) \mathrm{f} \rightarrow 6 \mathrm{p}^{2}$. The population level distribution and the corresponding temperatures were obtained using Boltzmann plots. The plasma electron densities were determined using well-known Stark broadening parameters of spectral lines. Special attention was dedicated to the possible self-absorption of the different transitions. Temporal evolution of the plasma parameters was studied between 0.1 and $9 \mu$ s. Stark broadening parameters of the spectral lines were measured at $2.5 \mu \mathrm{s}$ where the electron temperature was close to $11,300 \mathrm{~K}$ and the electron density to $0.8 \times 10^{16} \mathrm{~cm}^{-3}$. The experimental results obtained have been compared with the experimental and theoretical values given by other authors. A systematic trend of this parameter versus temperature of $4244.9 \AA \mathrm{Pb}$ II line has been presented.
\end{abstract}

\section{Introduction}

The application of laser ablation for chemical element analysis of solid sample is one of the most important applications of laser produced plasma (LPP) in science and technology [1,2]. Stark broadening data for a large number of transitions in many atomic and ionic spectra are needed not only for atomic structure research, but also for applications to astrophysics and analytical techniques. Laser produced plasma is one of the habitual methods for the experimental determination of different atomic and molecular parameters and in particular of the Stark broadening parameters. For that, LPP is one of the most good options as light source. We have already presented works in this way [1-5].

$\mathrm{Pb}$ II has been detected in several stars with the Goddard High Resolution Spectrograph (GHRS) aboard the Hubble Space Telescope (HST) [6], and the resonance lines 1682.1 $\AA$ and
$1433.9 \AA$ of $\mathrm{Pb}$ II are accessible in the GHRS spectral range (1150-3100 §). Also lead presents a large solar abundance [7]. Therefore, reliable $\mathrm{Pb}$ II Stark broadening profiles are needed to derive various astrophysical parameters. Experimental values might be preferable to theoretical ones but in the spectrum of the $\mathrm{Pb} I \mathrm{I}$ ion there are several intense lines for which no data at all can be found in the literature.

Miller et al. [8] obtained experimental data of seven visible $\mathrm{Pb}$ II lines at 11,600 K, adjusted to an electron density of $10^{17} \mathrm{~cm}^{-3}$. The data have an estimated relative uncertainty of about 30\%. Lakicevic [9] estimated the Stark half-widths for the $16,821 \AA$ resonant line of $\mathrm{Pb}$ II from systematic trends of Stark parameters versus inverse value of the lower level ionization potential.

Stark half-widths and shifts measured (within $\pm 15 \%$ ) for three spectral lines of Pb II (4248.3, 5545.7 and 5610.4 $\AA$ wavelengths in vacuum) were obtained at two different values of the electron temperature and density by Puric et al. [10]. Semiempirical values for the 5545.7 and $5610.4 \AA$ lines (wavelengths in vacuum) using the Coulomb approximation were also 


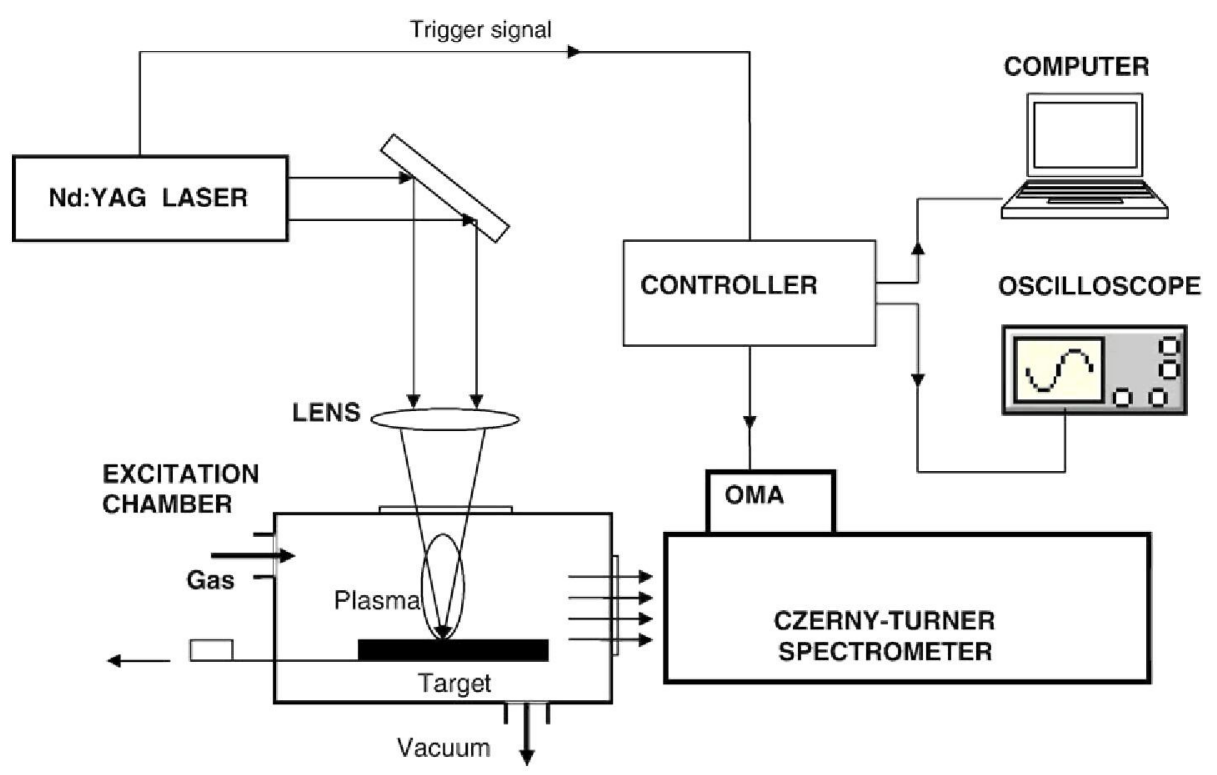

Fig. 1. Schematic of the experimental setup used for optical emission spectroscopic studies.

presented in this work. Fishman et al. [11] determined experimental values for three visible lines $(4388.1,4246.3$ and $6661.8 \AA$ - wavelengths in vacuum) using an impulsive capillary light source and confirmed the values obtained by these authors in earlier work [12].

Semiempirical calculations of Stark widths for 43 lines of $\mathrm{Pb}$ II arising from $n \mathrm{~s}^{2} \mathrm{~S}_{1 / 2}, n \mathrm{p}^{2} \mathrm{P}_{1 / 2,3 / 2}, n \mathrm{~d}^{2} \mathrm{D}_{3 / 2,5 / 2}$, and $5 \mathrm{f}^{2} \mathrm{~F}_{5 / 2,7 / 2}$ levels of $\mathrm{Pb} \mathrm{II}$ were performed in recent works by Colón and Alonso-Medina $[13,14]$. Stark widths were presented as functions of temperature for an electron density of $10^{17} \mathrm{~cm}^{-3}$.

In the present experimental work the LPP emission on a lead surface is used as light source to determine experimental Stark width of $31 \mathrm{~Pb}$ II lines between 1900 and $7000 \AA$ A. Stability and homogeneity of electron density and temperature in the plasma are determined by means of a study of the temporal evolution in different environmental conditions and target composition. The Local Thermodynamic Equilibrium (LTE) assumption is discussed by the analysis of these experimental working conditions. The values obtained are compared with existing experimental and theoretical values. We will describe in Section 2 the experimental system used for LPP. The results obtained are in Section 3, and the conclusions are in Section 4.

\section{Experimental setup}

The experimental system has been described in previous papers [1-5]. A schematic diagram of the experimental setup is present in Fig. 1.

A chamber was used to generate the plasma, in a vacuum or in a gas atmosphere. A vacuum of $10^{-5}$ Torr had also been attained inside the chamber by means of a turbomolecular pump, and it was filled with argon and maintained at a constant pressure of 6 Torr throughout the measurement, using a small continuous flow of gas to maintain the purity of the atmosphere. In this way the temperature, the electron density and the temporal evolution of LPP could be controlled. Fig. 2 presents a section of typical spectra.
Samples were located inside the chamber, on top of a device capable of moving it horizontally with respect to the laser beam, focused in such a way that the plasma was formed in each measurement on the smooth surface of the target and not on the center formed during the previous measurement.

The laser beam used to generate the plasma was produce by a Q-switched Nd:YAG laser (Quantel YG585), that generates $275 \mathrm{~mJ}$ pulses of $10 \mathrm{~ns}$ duration at a frequency of $20 \mathrm{~Hz}$ and $10,640 \AA$ wavelength. Its beam was focused to the sample by a

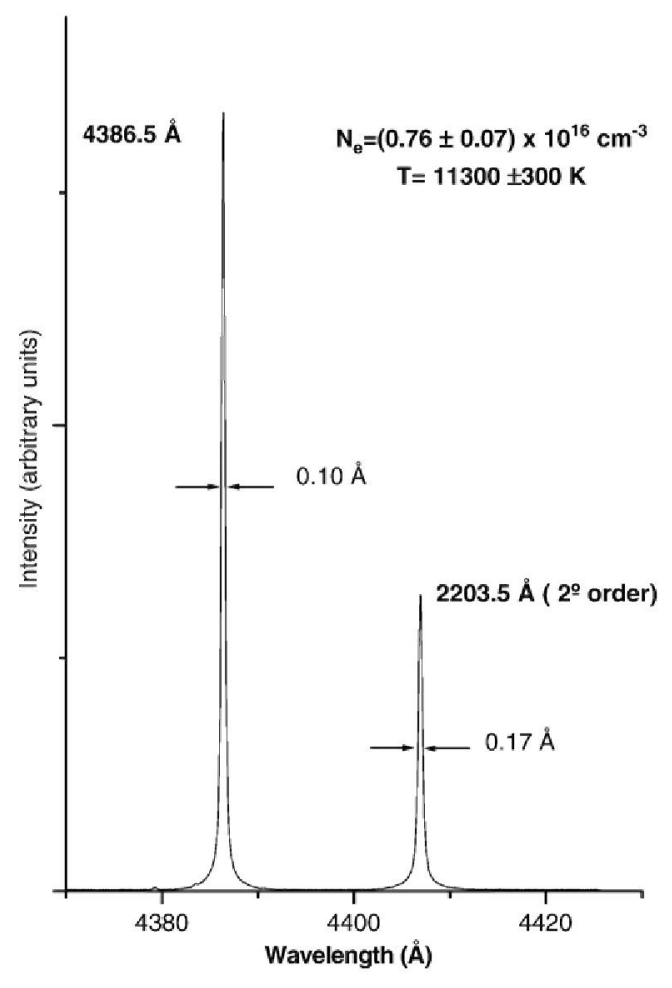

Fig. 2. Emission lines of $\mathrm{Pb}$ II at 6 Torr in argon ( $2.5 \mu$ s delay time). The FWHM are marked. 
lens of a focal distance of $12 \mathrm{~cm}$. The laser irradiance on the blank was $1.4 \times 10^{10} \mathrm{~W} \mathrm{~cm}^{-2}$, producing craters with the standard diameter of $0.5 \mathrm{~mm}$. The spatial width of the focused laser beam was measured by recording its image with a 1024-element linear silicon diode array. The light emitted by the LPP was transmitted, through a sapphire window, to the input slit of a $1 \mathrm{~m}$ Czerny-Turner spectrometer provided with a 2400 grooves $\mathrm{mm}^{-1}$ holographic grating. The resolution of the spectroscopic system was $0.3 \AA$ in the first order.

The spectral response of the system was obtained in the $1900-7000 \AA$ wavelength range by means of previously calibrated lamps. A deuterium lamp was used for the 1900-4000 $\AA$ range and a tungsten lamp was used for the $3500-7000 \AA$ range. The estimated error of this measurement was about $3 \%$. The calibration was also verified by means of Ar I and Ar II branching ratios to permit the comparison of the response selected in the spectral regions centered in 2500,3800 and $6500 \AA$ [2]. In order to obtain a statistical uncertainty of $3 \%$ various emission spectra were obtained and analyzed.

Spectra were recorded by a time-resolved optical multichannel analyzer (OMA III EG\&G) that allows the recording of spectra at a preset delay from the laser pulse and with a selected time length. Spectra were obtained at $0.1-9.0 \mu$ s delay from the laser pulse and light was collected during $0.1 \mu$ s in synchronism with the electronic trigger of the laser Q-switch. In each data acquisition period a correction was made with regard to the dark signal in the absence of the laser plasma.

The optical device contribution to the total broadening of the spectral line (instrumental profile) was determined by observation of various narrow lines emitted by hollow cathode lamps with a precision of $97 \%$, the instrumental full width at half maximum (FWHM) being $0.11 \AA$ for a wavelength of $3000 \AA$.

In order to take into account the possible self-absorption for the spectral lines, two different kinds of samples were used in the present experiment. One of lead (99.99\% purity) and others of tin-lead with a lead content of $75 \%, 25 \%, 5 \%$ and $0.5 \%$.

The analysis of the spectral lines was made by fitting the observed line shapes to numerically generated Voigt profiles. Local profiles were obtained after Abel inversion of the integrated intensity [15]. The fitting of the observed profiles provides the total intensity very accurately, as well as the broadening of the spectral lines.

The same experimental system was used to study the homogeneity of the plasma but, in order to have spatial resolution, the light was focused by means of a lens on a $1 \mathrm{~mm}$ light guide being able to select the point of the plasma from which the light emission was observed. The measurements were taken by scanning the plasma emission in two perpendicular directions, as can be seen in Fig. 1 of Ref. [5], to determine where the different atomic species of lead are located in the plasma and to determine the real values of the parameters of the plasma.

The population distribution of the levels and the corresponding temperatures were obtained using Boltzmann plots. The plasma electron densities were determined from the Stark broadening of well-known spectral lines. The existence of possible self-absorption effects on the measured $\mathrm{Pb}$ II lines was estimated taking into account the temperature and the electron density of the plasma.

\section{Experimental results}

\subsection{Emission spectrum}

The LPP emission spectrum was recorded in vacuum and in an argon atmosphere for different delay times. As a general rule, in the first times of the evolution of the plasma, the spectrum lines appear widened, and it is hard to distinguish them from the intense bremsstrahlung continuum emission for times of approximately $0.1 \mu \mathrm{s}$. For a time of approximately $0.4 \mu \mathrm{s}$ after the laser pulse, the species observed are the ionized atoms with high line intensities and widths. For longer times the widths and intensities of the neutral and single ionized species decrease considerably.

In Fig. 3 time-resolved emission spectra at $0.2-2.5 \mu \mathrm{s}$ from laser produced plasma are presented. In the vacuum the temporal evolution of the plasma is very fast. In argon atmosphere, at short times the spectral emission lines of Ar I, Ar II, Ar III, Pb I, Pb II and $\mathrm{Pb} \mathrm{III}$ appear. But the $\mathrm{Pb} \mathrm{II}$ emission lines appear at very early times when the temperature of the plasma is higher, disappearing at $0.9 \mu \mathrm{s}$. Practically at $2.5 \mu \mathrm{s}$ there is no presence

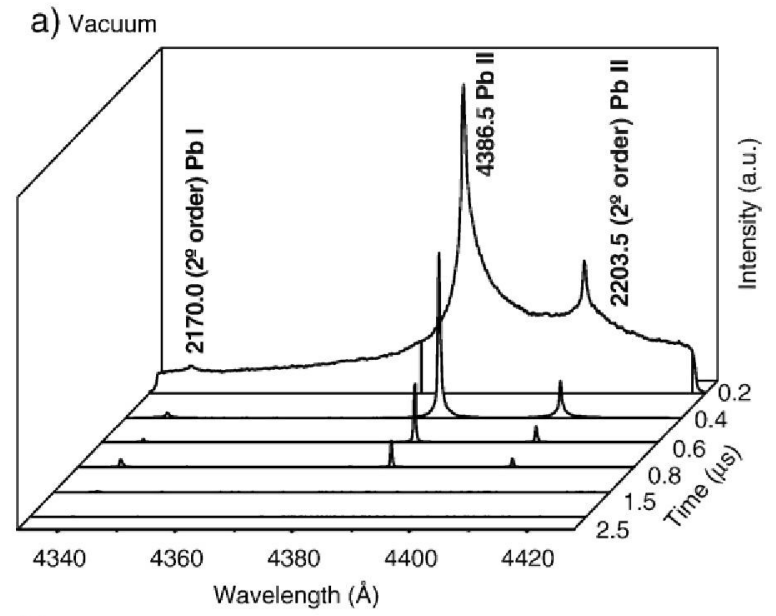

b) 6 Torr Ar

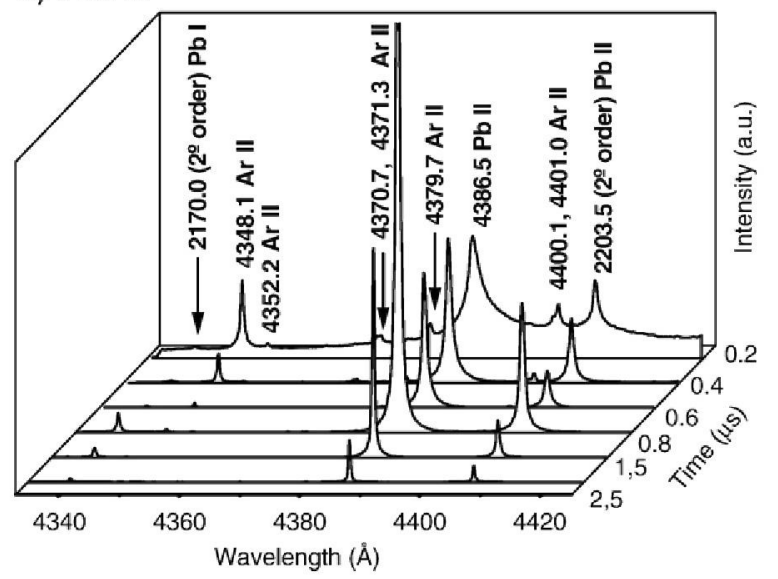

Fig. 3. Time-resolved emission spectra section from laser-produced lead plasma in: a) vacuum and b) 6 Torr in argon. 


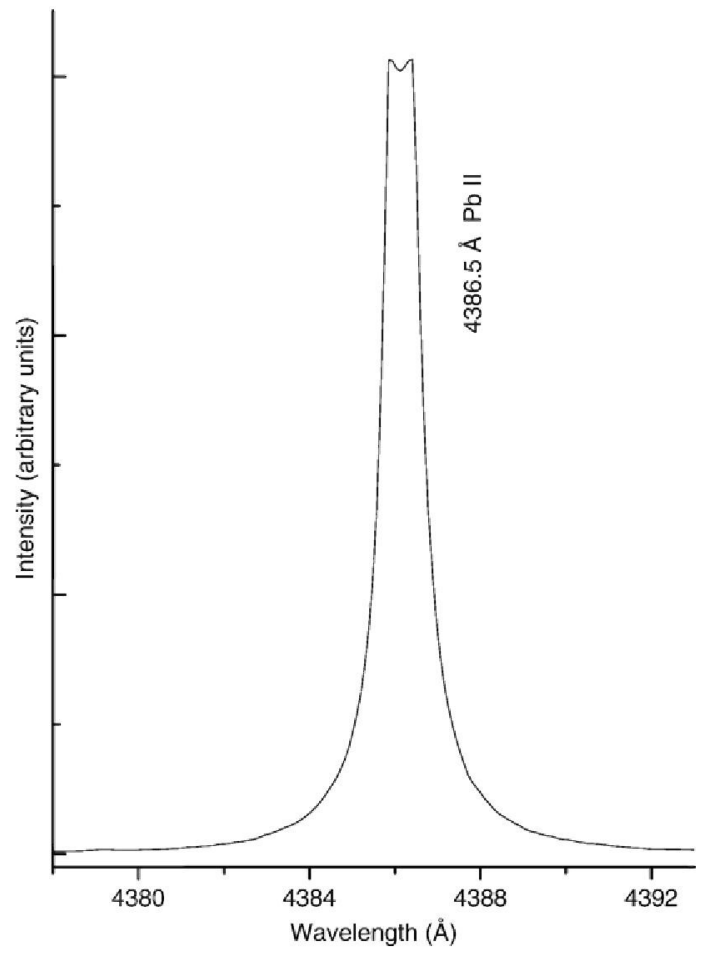

Fig. 4. Emission line of $4386.5 \AA$ of $\mathrm{Pb}$ II at 6 Torr in argon at $0.8 \mu$ s delay time

of argon emission lines. The intensities of the emission lines corresponding to the $\mathrm{Pb} \mathrm{I}$ and $\mathrm{Pb}$ II reach maximum values at $0.8 \mu$ s. Clearly the self-absorption effects can be appreciated, as can be seen for instance in the $4386.5 \AA \mathrm{Pb}$ II line in Fig. 4. From $0.9 \mu$ s the self-absorption effects disappear.

In our experimental conditions - with a delay time of $2.5 \mu \mathrm{s}$ after the laser pulse, in an atmosphere of argon at 6 Torr - all the transitions of the $\mathrm{Pb}$ II spectrum can be observed. Under these conditions none of the studied lines presents self-absorption effects, except for the $2203 \AA$ line. This line is an object of a separate study. Relative intensities have been measured for 31 lines of $\mathrm{Pb}$ II, evaluating the area under each one, for which purpose adjustments were made to local profiles of the lines by means of a convolution of the instrumental profile, known, with the Voigt profiles obtained from the contributions selected, Lorentz and Gaussian. The setting of the profiles allows us to obtain the total intensities of the lines, as well as the Lorentz and Gaussian contribution in each line.

\subsection{Determination of the plasma temperature}

In optically thin plasma the relative intensities, $I_{i j}$ of the lines emitted from a given state of excitation can be used to calculate the electron temperature, if the $A_{i j}$ transition probabilities are known, by the expression

$I_{i j}=\frac{A_{i j} g_{i}}{U(T)} N \exp \left(\frac{-E_{i}}{k T}\right)$

for a transition from a higher state $i$ to a lower state $j, I_{i j}$ is the relative intensity, $E_{i}$ and $g_{i}$ are the energy and statistical weight of level $i, U(T)$ is the atomic species partition function, $N$ the total
Table 1

Parameters for electron temperature determination (in argon at a pressure of 6 Torr, delay time of $2.5 \mu \mathrm{s}$ )

\begin{tabular}{llcc}
\hline Transitions & $\lambda(\AA)$ & $E_{i}(\mathrm{eV})$ & $A_{i j}\left(\times 10^{6} \mathrm{~s}^{-1}\right)$ Ref. $[17]$ \\
\hline $8 \mathrm{~d}^{2} \mathrm{D}_{3 / 2} \rightarrow 7 \mathrm{p}^{2} \mathrm{P}_{1 / 2}$ & 3455.1 & 12.819 & $43.7 \pm 7.0$ \\
$9 \mathrm{~s}^{2} \mathrm{~S}_{1 / 2} \rightarrow 7 \mathrm{p}^{2} \mathrm{P}_{3 / 2}$ & 4152.8 & 12.566 & $22.6 \pm 3.6$ \\
$7 \mathrm{~d}^{2} \mathrm{D}_{3 / 2} \rightarrow 7 \mathrm{p}^{2} \mathrm{P}_{1 / 2}$ & 5042.6 & 11.690 & $101.7 \pm 15.2$ \\
$5 \mathrm{f}^{2} \mathrm{~F}_{5 / 2} \rightarrow 6 \mathrm{~d}^{2} \mathrm{D}_{3 / 2}$ & 4386.5 & 11.473 & $147.1 \pm 14.7$ \\
$7 \mathrm{p}^{2} \mathrm{P}_{3 / 2} \rightarrow 7 \mathrm{~s}^{2} \mathrm{~S}_{1 / 2}$ & 5608.9 & 9.581 & $83.1 \pm 8.3$ \\
\hline
\end{tabular}

density of emitting atoms, $k$ the Boltzmann constant and $T$ the temperature. If we were to plot $\ln \left(I_{i j} / g_{i} A_{i j}\right)$ vs. $E_{i}$, the Boltzmann plot, the resulting straight line would have a slope of $-1 / k T$, and therefore the temperature can be obtained without having to know the total density of atoms or the atomic species partition function. The energies of the different levels are those of Moore [16,17].

The designations and the $\mathrm{Pb}$ II transition probabilitics selected for determination of the excitation temperature by the Boltzmann plot are shown in Table 1 . The relative intensities $I_{i j}$ required for application of this method were obtained in this study and the corresponding transition probabilities are those obtained using a hollow cathode lamp in a previous study [18].

Fig. 5 displays a Boltzmann plot from which a value of $11,300 \pm 300 \mathrm{~K} \Delta E=3.328 \mathrm{eV}$ was obtained from the electron temperature.

\subsection{Determination of the plasma electron density}

The electron density, $N_{\mathrm{e}}$, of the plasma investigated has been obtained by comparing the Stark broadenings for several transitions with those of other authors, see Table 2, using the expression by Milosavljevic and Poparic [19]:

$\omega=\omega_{p}\left(\frac{N_{e}^{2}}{10^{16}}\right)\left[1+1.75 A\left(\frac{N_{\mathrm{e}}}{10^{16}}\right)^{1 / 4}\left(1-1.2 N_{\mathrm{D}}^{-1 / 3}\right)\right]$

where $\omega$ is the full width at half maximum (FWHM) of the transition considered, $\omega_{\mathrm{p}}$ is the electron impact, full half-width, parameter, $A$ is the ion broadening parameter, and $N_{\mathrm{D}}$ is the number of particles in the Debye sphere, which must be in

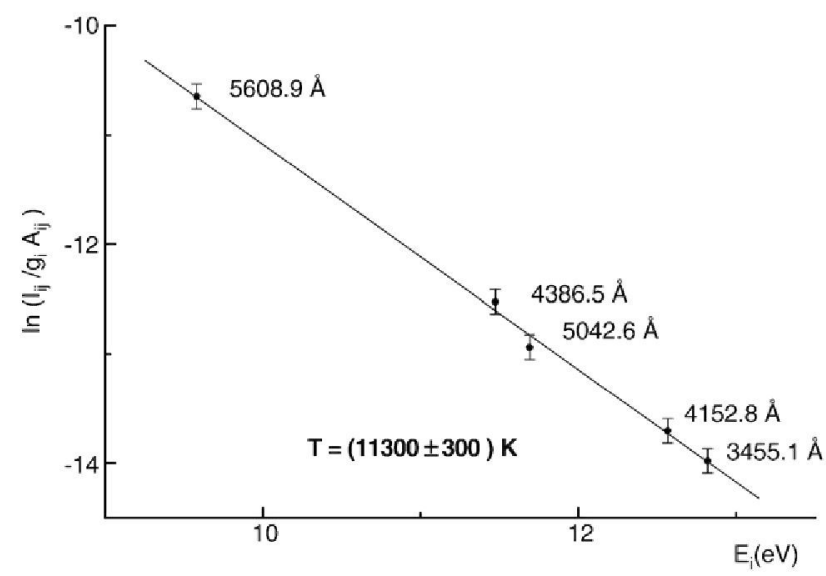

Fig. 5. Boltzmann plot for $\mathrm{Pb}$ II lines from laser-produced lead plasma at $2.5 \mu \mathrm{s}$ delay time in argon at a pressure of 6 Torr. 
Table 2

Electron density of laser-produced lead plasma (6 Torr in argon, delay time of $2.5 \mu \mathrm{s}$ ) estimated from the Stark width of several $\mathrm{Pb} I I$ lines

\begin{tabular}{lllll}
\hline Pb II transition & $\lambda(\AA)$ & $\begin{array}{l}\text { Temperature } \\
\left(\times 10^{3} \mathrm{~K}\right)\end{array}$ & $\begin{array}{l}N_{\mathrm{e}}=10^{17} \mathrm{~cm}^{-3} \\
\omega_{\text {exp }}(\AA)(\mathrm{FWHM})\end{array}$ & $\begin{array}{l}N_{\mathrm{e}}\left(\times 10^{16}\right) \\
\mathrm{cm}^{-3}\end{array}$ \\
\hline $7 \mathrm{~d}^{2} \mathrm{D}_{3 / 2} \rightarrow 7 \mathrm{p}^{2} \mathrm{P}_{1 / 2}$ & 5042.6 & 11.6 & $3.84^{\mathrm{a}}( \pm 20 \%)$ & $0.87 \pm 20 \%$ \\
$7 \mathrm{~d}^{2} \mathrm{D}_{5 / 2} \rightarrow 7 \mathrm{p}^{2} \mathrm{P}_{3 / 2}$ & 5544.3 & 11.6 & $3.60^{\mathrm{a}}( \pm 20 \%)$ & $0.84 \pm 25 \%$ \\
& & 16.0 & $3.18^{\mathrm{b}}( \pm 15 \%)$ & $0.84 \pm 20 \%$ \\
$5 \mathrm{f}^{2} \mathrm{~F}_{5 / 2} \rightarrow 6 \mathrm{~d}^{2} \mathrm{D}_{3 / 2}$ & 4386.5 & 11.6 & $1.72^{\mathrm{a}}( \pm 20 \%)$ & $0.76 \pm 25 \%$ \\
$5 \mathrm{f}^{2} \mathrm{~F}_{7 / 2} \rightarrow 6 \mathrm{~d}^{2} \mathrm{D}_{5 / 2}$ & 4244.9 & 11.6 & $1.69^{\mathrm{a}}( \pm 20 \%)$ & $0.71 \pm 25 \%$ \\
& & 16.0 & $1.52^{\mathrm{b}}( \pm 15 \%)$ & $0.73 \pm 20 \%$ \\
$5 \mathrm{f}^{2} \mathrm{~F}_{7 / 2} \rightarrow 6 \mathrm{p}^{2}{ }^{4} \mathrm{P}_{5 / 2}$ & 5372.3 & 11.6 & $2.27^{\mathrm{a}}( \pm 20 \%)$ & $0.79 \pm 25 \%$ \\
\hline${ }^{\mathrm{a}}$ Miller et al. [8]. & & & & \\
$\mathrm{b}$ Puric et al. [10]. & & &
\end{tabular}

excess of the lower limit $N_{\mathrm{D}}=2$ of the Debye approximation for correlation effects. In our measurements we have assumed that $A$ is negligible [20].

We have selected lines with broadening Stark widths published that present small uncertainties: $5042.6 \AA, 5544.3 \AA$, $4386.5 \AA, 4244.9 \AA$ and $5372.3 \AA \mathrm{Pb}$ II lines. The fourth column displays the Stark broadening measured by Miller et al. [8] and Puric et al. [10]. The fifth column displays the electron densities obtained using the values of the previous column, normalized to the temperature, $11,300 \mathrm{~K}$, of our plasma.

A plasma density of $0.76 \times 10^{-16} \mathrm{~cm}^{-3}$ with an uncertainty of about $8 \%$ can be estimated for our study.

McWhirte's criterion, for the lower limit of the electron density, has been used to support the LTE hypothesis, using the following expression.

$N_{e}^{2}\left(\mathrm{~cm}^{-3}\right) \geq 1.6 \times 10^{12} \sqrt{T}(\Delta E)^{3}$

where $\Delta E$, in $\mathrm{eV}$, is the energy difference between the upper and lower states, and $T$, in $\mathrm{K}$, the temperature and $N_{\mathrm{e}}$ the lower limit of the electron density necessary to maintain the populations of the energy levels at $10 \%$ of the LTE. Using the values obtained of the lines of $\mathrm{Pb} \mathrm{II}$, the critical $N_{\mathrm{e}}$ is $0.626 \times 10^{-16} \mathrm{~cm}^{-3}$.

\subsection{Self-absorption analysis}

With the aforementioned values of $N_{\mathrm{e}}$ and $T$ we can calculate the absorption coefficient for the studied lines, using the following equation [21], expressed in $\mathrm{m}^{-1}$ :

$k_{\omega}=\frac{\pi_{e}^{2}}{2 \varepsilon_{0} m c} f_{i k} N_{i}\left[1-\frac{N_{k} g_{i}}{N_{i} g_{k}}\right] g(\omega)$

$f_{i k}$ is the oscillator strength (absorption) and $g(\omega)$ is the normalized profile of the line. In the maximum, $\omega=0$, and for a Lorentz profile, $g(o)=2 / \pi \Gamma$, where $T$ is the FWHM of the line.

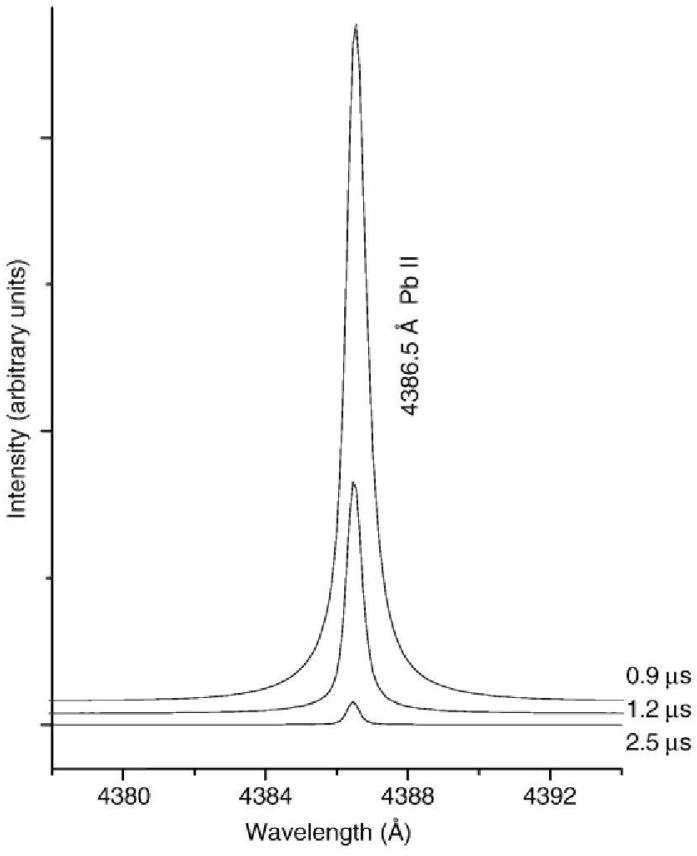

Fig. 6. Emission line of $4386.5 \AA$ of $\mathrm{Pb} I I$ at 6 Torr in argon. At $0.9,1.2$ and $2.5 \mu$ delay times from the laser pulse.

A line may be considered optically thin if $k_{\omega} L \leq 0.05$ [22]. In our experimental conditions the value of the optical depth, $k_{w} L$, is not in excess of 0.02 (for example 0.019 in $6660.0 \AA, 0.006$ in $5042.6 \AA$ ) for all the lines studied with the exception of the line $2203.53 \AA$ that presents a value of 0.09 . A special study for this line has been carried out using like sample different alloys of lead and tin with falling contents in lead. This study is present in Table 3.

\subsection{Spatial and temporal evolution of temperature and electron density}

The values given for $N_{\mathrm{e}}$ and $T$ correspond to the center of plasma. To determine the change of these parameters in different regions, we have obtained their values at different points using various lines of $\mathrm{Pb}$ II. We have obtained that the plasma in argon atmosphere is homogeneous for $N_{\mathrm{e}}$ and $T$. Deviations from the average are less than $15 \%$ for $N_{\mathrm{e}}$ and $5 \%$ for $T$ in a region measuring approximately $2 \mathrm{~mm}$ in size corresponding to $95 \%$ of the light emission. Similar results can be seen in Refs. [1-3].

Temperatures and electron densities of plasma have been obtained for $0.9 \mu \mathrm{s}, 1.2 \mu \mathrm{s}$ and $2.5 \mu \mathrm{s}$ delay times from the laser pulse using the target with $99.99 \%$ lead. In Fig. 6, a typical section of emission spectra corresponding to the $4386.5 \AA \mathrm{Pb}$ II line for these delay times is presented. In this figure, it can be seen that self-absorption effects have disappeared.

Table 3

Optical depth, $k_{\omega} L$, study of the $2203.5 \AA \mathrm{Pb}$ II emission line in different targets

\begin{tabular}{|c|c|c|c|c|c|}
\hline $\begin{array}{l}\text { Target } \\
N_{e} \sim 10^{16} \mathrm{~cm}^{-3}\end{array}$ & $\begin{array}{l}99.99 \% \mathrm{~Pb} \\
T=11,300 \mathrm{~K}\end{array}$ & $\begin{array}{l}75 \% \mathrm{~Pb} \\
T=11,300 \mathrm{~K}\end{array}$ & $\begin{array}{l}25 \% \mathrm{~Pb} \\
T=11,100 \mathrm{~K}\end{array}$ & $\begin{array}{l}5 \% \mathrm{~Pb} \\
T=11,000 \mathrm{~K}\end{array}$ & $\begin{array}{l}0.5 \% \mathrm{~Pb} \\
T=11,000 \mathrm{~K}\end{array}$ \\
\hline$k_{\omega} L$ & 0.09 & 0.05 & 0.04 & 0.02 & 0.009 \\
\hline
\end{tabular}


Table 4

Temperatures and electron densities of plasma for $0.9 \mu \mathrm{s}, 1.2 \mu \mathrm{s}$ and $2.5 \mu \mathrm{s}$ delay times from the laser pulse

\begin{tabular}{lccc}
\hline$\lambda(\AA)$ & Upper level energy in eV & $g_{i}$ & $A_{i j}\left(\times 10^{6} \mathrm{~s}^{-1}\right)$ Refs. [2,18] \\
\hline 5163.3 & 9.58 & 4 & $1.6 \pm 0.2$ \\
4386.5 & 11.47 & 6 & $147.1 \pm 14.7$ \\
3713.9 & 12.92 & 6 & $41.9 \pm 6.2$ \\
& & & $N_{\mathrm{e}}\left(\times 10^{16} \mathrm{~cm}^{-3}\right)$ \\
Delay time ( $\mu \mathrm{s})$ & Temperature $(\mathrm{K})$ & $0.76 \pm 0.15$ \\
\hline 2.5 & $11300 \pm 300$ & $1.60 \pm 0.35$ \\
1.2 & $14500 \pm 420$ & $3.0 \pm 0.6$ \\
0.9 & $15300 \pm 460$ &
\end{tabular}

Additional spectroscopic data of $\mathrm{Pb}$ II were employed for temperature determination.

Temperatures have been measured from the Boltzmann plot, using the $3713.9,4386.5$ and $5163.3 \AA$ cmission lincs. Elcctron densities were obtained using the experimental Stark broadening of $4386.5 \AA$ and the broadening Stark parameter of Miller et al. [8]. Results are given in Table 4.

The experimental dependence of Stark width, $\omega$, on the electron temperature for the $4244.9 \AA$ line of $\mathrm{Pb}$ II has been studied, in the above mentioned delay times, in order to test our procedure and to complete the experimental data of other authors. Results are presented in Table 5 and are displayed together with the values of the other authors, normalized to an electron density of $1.0 \times 10^{16} \mathrm{~cm}^{-3}$, in Fig. 7. As can be appreciated in this figure the results obtained in this work are in good agreement with the experimental trend of the results obtained previously by other authors.

\subsection{Pb II Stark broadening parameter}

In this work the spectroscopic analysis of the laser produced plasma emission provides experimental Stark broadening parameters for 31 lines of $\mathrm{Pb}$ II. This data includes new values for 23 emission lines.

Table 6 shows the results of our experimental data of full width half maximum (FWHM) parameters normalized to an electron density of $10^{16} \mathrm{~cm}^{-3}$. The corresponding errors include uncertainties in the instrumental profile and statistical errors after an average of several spectra with a total of about 100 laser shots. The possible error due to the experimental uncertainty in the density of electrons in this study is not included. The first three columns denote the corresponding transition array, the multiplet and the wavelengths (in $\AA$ ) for each studied transition. Electron temperatures Stark are indicated in the fourth column. The fifth column compiles our experimental Stark parameters.

Table 5

Experimental Stark FWHM, $\omega(\AA)$, dependence on the electron temperature scaled to the electron density of a $1.0 \times 10^{16} \mathrm{~cm}^{-3}$ for the $4244.9 \AA$ line of $\mathrm{Pb} \mathrm{II}$

\begin{tabular}{lll}
\hline Delay times $(\mu \mathrm{s})$ & Temperature $(\mathrm{K})$ & $\omega(\AA)$ \\
\hline 2.5 & $11,300 \pm 300$ & 0.190 \\
1.2 & $14,500 \pm 420$ & 0.152 \\
0.9 & $15,300 \pm 460$ & 0.150 \\
\hline
\end{tabular}

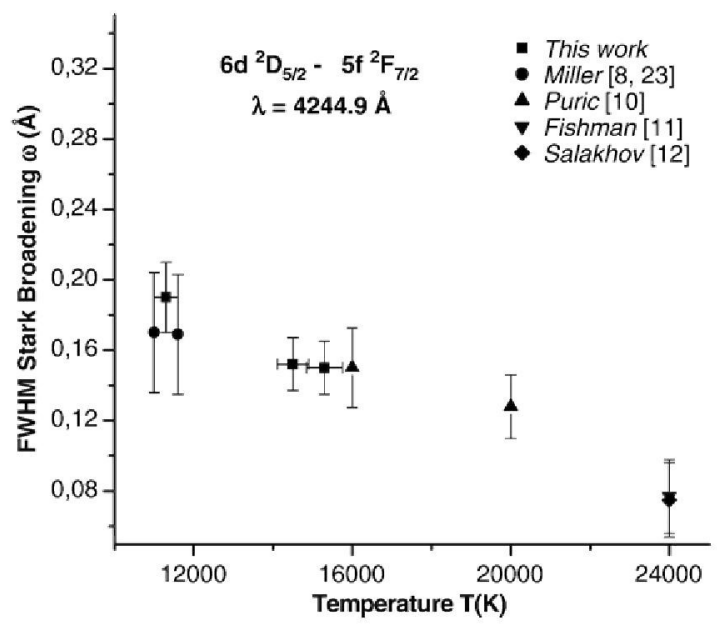

Fig. 7. Temperature dependence of Stark width of $4244.9 \AA \mathrm{Pb}$ II line scaled to the electron density of $1 \times 10^{16} \mathrm{~cm}^{-3}$.

In the sixth column the experimental values of other authors are displayed $[8,10-12,23,24]$. In the seventh column we present our theoretical values calculated in a previous work [13].

The $2203.5 \AA \mathrm{Pb}$ II line was measured using a $0.5 \%$ lead purity sample, in the experimental conditions related in Table 3 , in order to eliminate the self-absorption effects.

As can be easily seen, all the experimental data determined in this work agree with the previously available, with the exception of the one presented by Djenize et al. [24] for the $2203.5 \AA$ line. In this case a remarkable difference exists.

Theoretical data were calculated by ourselves in 2002 [13]. As can be seen and as can be expected these theoretical data agree with the experimental results obtained in the present work, with the exception of certain discrepancies (in the lines that involve the 5 f configuration) whose origin was studied in the above mentioned reference. Nevertheless a remarkable discrepancy between our $2203.5 \AA$ line experimental value and our theoretical values exists. A more itemized theoretical study of the Stark broadening of this line is necessary.

\section{Conclusions}

Optical emission spectroscopy is used to characterize the laser produced plasma from several lead targets in vacuum and argon atmosphere. Time- and space-resolved measurements of electron density and temperature have been carried out by using the Boltzmann plot of line intensities of several $\mathrm{Pb}$ II transitions and the experimental well-known Stark broadening profile of the $4386.5 \AA$ line $\mathrm{Pb} \mathrm{II.} \mathrm{Self-absorption} \mathrm{analysis} \mathrm{of} \mathrm{the} \mathrm{tran-}$ sitions involved in this work has been carried out for several targets. An itemized study of temperature dependence of the Stark broadening corresponding to the $4244.9 \AA$ line of $\mathrm{Pb}$ II was carried out in order to test our method. A good agreement with the experimental data was obtained. Spectroscopic analysis of the plasma light emission provided us the experimental Stark width of 31 emission lines of $\mathrm{Pb} \mathrm{II}$. Only data for 8 lines are previously referenced. A good agreement with previously referenced data was found in all cases. 
Table 6

Experimental Stark FWHM, $\omega(\AA)$, of emission lines of Pb II normalized at $N_{\mathrm{e}}=10^{16} \mathrm{~cm}^{-3}$ (6 Torr of argon, delay time of $2.5 \mu \mathrm{s}$ )

\begin{tabular}{|c|c|c|c|c|c|c|}
\hline \multirow{3}{*}{$\begin{array}{l}\text { Transition } \\
\text { array }\end{array}$} & \multirow[t]{3}{*}{ Multiplet } & \multirow[t]{3}{*}{$\lambda(\AA)$} & \multirow{3}{*}{$\begin{array}{l}\text { Temperature } \\
\left(\times 10^{3} \mathrm{~K}\right)\end{array}$} & \multicolumn{3}{|l|}{$\omega(\AA)$} \\
\hline & & & & \multirow[t]{2}{*}{ This work } & \multicolumn{2}{|l|}{ Other works } \\
\hline & & & & & Experiment & Theory \\
\hline \multirow[t]{2}{*}{$6 p-7 s$} & ${ }^{2} \mathrm{P}_{3 / 2}-{ }^{2} \mathrm{~S}_{1 / 2}$ & 2203.5 & 11.3 & $0.11 \pm 0.01$ & & $0.0125^{\mathrm{a}}$ \\
\hline & & & 28.0 & & $0.0049^{\mathrm{b}}$ & \\
\hline $7 p-8 s$ & ${ }^{2} \mathrm{P}_{1 / 2}-\mathrm{S}_{1 / 2}$ & 6791.2 & 11.3 & $0.22 \pm 0.02$ & & $0.342^{\mathrm{a}}$ \\
\hline \multirow[t]{3}{*}{$7 p-9 s$} & ${ }^{2} \mathrm{P}_{1 / 2}-{ }^{2} \mathrm{~S}_{1 / 2}$ & 3718.3 & 11.3 & $0.33 \pm 0.03$ & & $0.268^{\mathrm{a}}$ \\
\hline & ${ }^{2} \mathrm{P}_{3 / 2}-{ }^{2} \mathrm{~S}_{1 / 2}$ & 4152.8 & 11.3 & $0.32 \pm 0.04$ & & $0.387^{\mathrm{a}}$ \\
\hline & & & 11.6 & & $0.234 \pm 35 \%$ & \\
\hline \multirow[t]{2}{*}{$7 p-10 s$} & ${ }^{2} \mathrm{P}_{1 / 2}-{ }^{2} \mathrm{~S}_{1 / 2}$ & 2986.9 & 11.3 & $0.21=0.02$ & & $0.504^{\mathrm{a}}$ \\
\hline & ${ }^{2} \mathrm{P}_{3 / 2}-{ }^{2} \mathrm{~S}_{1 / 2}$ & 3260.9 & 11.3 & $0.17 \pm 0.03$ & & $0.626^{\mathrm{a}}$ \\
\hline \multirow[t]{8}{*}{$7 s-7 p$} & ${ }^{2} \mathrm{~S}_{1 / 2}-{ }^{2} \mathrm{P}_{1 / 2}$ & 6660.0 & 11.3 & $0.12=0.01$ & & $0.170^{\mathrm{a}}$ \\
\hline & & & 24.0 & & $0.09 \pm 35 \% \%^{\mathrm{d}}$ & \\
\hline & & & 24.0 & & $0.09 \pm 35 \%$ & \\
\hline & ${ }^{2} \mathrm{~S}_{1 / 2}-{ }^{2} \mathrm{P}_{3 / 2}$ & 5608.9 & 11.3 & $0.14 \pm 0.02$ & & $0.197^{\mathrm{a}}$ \\
\hline & & & 11.3 & $0.14 \pm 0.02$ & $0.20 \pm 35 \%{ }^{\mathrm{f}}$ & \\
\hline & & & 11.6 & & $0.196 \pm 35 \%{ }^{\mathrm{c}}$ & \\
\hline & & & 16.0 & & $0.21 \pm 15 \%^{g}$ & \\
\hline & & & 20.0 & & $0.17 \pm 15 \% \%^{g}$ & \\
\hline \multirow[t]{2}{*}{$6 p^{2}-7 p$} & ${ }^{4} \mathrm{P}_{1 / 2}-{ }^{2} \mathrm{P}_{1 / 2}$ & 6041.4 & 11.3 & $0.16 \pm 0.03$ & & \\
\hline & ${ }^{4} \mathrm{P}_{1 / 2}-{ }^{2} \mathrm{P}_{3 / 2}$ & 5163.3 & 11.3 & $0.19 \pm 0.02$ & & \\
\hline \multirow[t]{2}{*}{$7 s-8 p$} & ${ }^{2} \mathrm{~S}_{1 / 2}-{ }^{2} \mathrm{P}_{1 / 2}$ & 2805.9 & 11.3 & $0.076 \pm 0.01$ & & $0.123^{\mathrm{a}}$ \\
\hline & ${ }^{2} \mathrm{~S}_{1 / 2}-{ }^{2} \mathrm{P}_{3 / 2}$ & 2717.4 & 11.3 & $0.20 \pm 0.03$ & & $0.208^{\mathrm{a}}$ \\
\hline \multirow{2}{*}{$6 p^{2}-8 p$} & ${ }^{4} \mathrm{P}_{1 / 2}-{ }^{2} \mathrm{P}_{1 / 2}$ & 3945.7 & 11.3 & $0.19 \pm 0.03$ & & \\
\hline & ${ }^{4} \mathrm{P}_{1 / 2}-{ }^{2} \mathrm{P}_{3 / 2}$ & 3665.5 & 11.3 & $0.17 \pm 0.02$ & & \\
\hline \multirow[t]{8}{*}{$7 p-7 d$} & ${ }^{2} \mathrm{P}_{1 / 2}-{ }^{2} \mathrm{D}_{3 / 2}$ & 5042.6 & 11.3 & $0.39=0.04$ & & $0.417^{\mathrm{a}}$ \\
\hline & & & 11.6 & & $0.384 \pm 20 \% \%^{\mathrm{c}}$ & \\
\hline & ${ }^{2} \mathrm{P}_{3 / 2}{ }^{-2} \mathrm{D}_{3 / 2}$ & 5876.6 & 11.3 & $0.36 \pm 0.03$ & & \\
\hline & ${ }^{2} \mathrm{P}_{3 / 2}-{ }^{2} \mathrm{D}_{5 / 2}$ & 5544.3 & 11.0 & $0.34 \pm 0.03$ & $0.360 \pm 20 \%{ }^{\mathrm{f}}$ & \\
\hline & & & 11.3 & & & $0.470^{\mathrm{a}}$ \\
\hline & & & 11.6 & & $0.360 \pm 20 \%$ & \\
\hline & & & 16.0 & & $0.328 \pm 15 \%$ & \\
\hline & & & 20.0 & & $0.210 \pm 15 \%^{g}$ & \\
\hline \multirow[t]{3}{*}{$7 p-8 d$} & ${ }^{2} \mathrm{P}_{1 / 2}-{ }^{2} \mathrm{D}_{3 / 2}$ & 3455.1 & 11.3 & $0.35 \pm 0.05$ & & \\
\hline & ${ }^{2} \mathrm{P}_{3 / 2}{ }^{-2} \mathrm{D}_{3 / 2}$ & 3827.2 & 11.3 & $0.25 \pm 0.04$ & & \\
\hline & ${ }^{2} \mathrm{P}_{3 / 2}{ }^{-2} \mathrm{D}_{5 / 2}$ & 3713.9 & 11.3 & $0.41 \pm 0.06$ & & \\
\hline \multirow[t]{12}{*}{$6 \mathrm{~d}-5 \mathrm{f}$} & ${ }^{2} \mathrm{D}_{3 / 2}{ }^{-2} \mathrm{~F}_{5 / 2}$ & 4386.5 & 11.3 & $0.13 \pm 0.02$ & & $0.270^{\mathrm{a}}$ \\
\hline & & & 11.6 & & $0.172 \pm 20 \%{ }^{\mathrm{c}}$ & \\
\hline & & & 20.0 & & $0.07 \pm 30 \%{ }^{\mathrm{d}}$ & \\
\hline & & & 27.0 & & $0.03^{\mathrm{b}}$ & \\
\hline & ${ }^{2} \mathrm{D}_{5 / 2-{ }^{-}}{ }^{2} \mathrm{~F}_{5 / 2}$ & 4242.1 & 11.3 & $0.19 \pm 0.02$ & & $0.234^{\mathrm{a}}$ \\
\hline & ${ }^{2} \mathrm{D}_{5 / 2}-{ }^{2} \mathrm{~F}_{7 / 2}$ & 4244.9 & 11.0 & & $0.17 \pm 20 \%{ }^{\mathrm{f}}$ & \\
\hline & & & 11.3 & $0.19 \pm 0.02$ & & $0.287^{\mathrm{a}}$ \\
\hline & & & 11.6 & & $0.169 \pm 20 \%{ }^{\mathrm{c}}$ & \\
\hline & & & 16.0 & & $0.152 \pm 15 \%{ }^{g}$ & \\
\hline & & & 20.0 & & $0.128 \pm 15 \%{ }^{\mathrm{g}}$ & \\
\hline & & & 24.0 & & $0.075 \pm 20 \% \%^{\mathrm{d}}$ & \\
\hline & & & 24.0 & & $0.077 \pm 20 \%{ }^{e}$ & \\
\hline $6 \mathrm{p}^{2}-5 f$ & ${ }^{4} \mathrm{P}_{1 / 2}-{ }^{2} \mathrm{~F}_{5 / 2}$ & 3785.9 & 11.3 & $0.18 \pm 0.03$ & & \\
\hline & ${ }^{4} \mathrm{P}_{5 / 2}-{ }^{2} \mathrm{~F}_{5 / 2}$ & 5367.6 & 11.3 & $0.22 \pm 0.02$ & & $0.392^{\mathrm{a}}$ \\
\hline & ${ }^{4} \mathrm{P}_{5 / 2}-{ }^{2} \mathrm{~F}_{7 / 2}$ & 5372.1 & 11.3 & $0.20 \pm 0.02$ & & $0.476^{\mathrm{a}}$ \\
\hline & & & 11.6 & & $0.227 \pm 20 \% \%^{\mathrm{c}}$ & \\
\hline $6 \mathrm{~d}-6 \mathrm{f}$ & ${ }^{2} \mathrm{D}_{3 / 2}-{ }^{2} \mathrm{~F}_{5 / 2}$ & 3016.4 & 11.3 & $0.28 \pm 0.03$ & & \\
\hline & ${ }^{2} \mathrm{D}_{5 / 2}-{ }^{2} \mathrm{~F}_{5 / 2}$ & 2947.4 & 11.3 & $0.32=0.03$ & & \\
\hline & ${ }^{2} \mathrm{D}_{5 / 2}-{ }^{2} \mathrm{~F}_{7 / 2}$ & 2948.5 & 11.3 & $0.24 \pm 0.02$ & & \\
\hline $6 p^{2}-6 f$ & ${ }^{4} \mathrm{P}_{3 / 2}-{ }^{2} \mathrm{~F}_{5 / 2}$ & 2719.8 & 11.3 & $0.11=0.02$ & & \\
\hline & ${ }^{4} \mathrm{P}_{5 / 2}-{ }^{2} \mathrm{~F}_{7 / 2}$ & 3451.7 & 11.3 & $0.30 \pm 0.04$ & & \\
\hline
\end{tabular}

\footnotetext{
a Colón and Alonso-Medina [13].

b Dejenize et al. [24].

c Miller et al. [8].

d Fishman et al. [11].

e Salakhov et al. [12].

${ }^{f}$ Miller et al. [23].

g Puric et al. [10].
}

\section{Acknowledgments}

This work has been supported by the project M0500204167 of the Spanish Universidad Politécnica de Madrid (support to the lines of UPM investigation groups, R05/1926). 